\title{
BRIEF INTRODUCTION TO SOME NEW PAPERS ON LATERAL TRANSFER RECONSTRUCTION, DRUG CANDIDATE SCREENING, DISEASE GENE IDENTIFICATION, AND OTHER RESULTS
}

This issue of the Journal of Bioinformatics and Computational Biology presents six research papers and two reviews. These articles are briefly summarized below.

Identification of lateral transfers in evolution is becoming an important problem in computational biology. Lateral transfers between distant taxa relative to a tree often result in large deviations from tree-like behavior. Such deviations violate the so-called Kalmanson inequalities that characterize the distance matrices of normal phylogenetic networks. In this issue, Thuillard and Moulton ${ }^{1}$ exploit this fact to develop a new approach to identify and reconstruct lateral transfers from distance matrices.

Recognition of the transmembrane domains and their topology has long been studied in computational biology. ${ }^{2-4}$ However, a more detailed understanding of how the correct spatial arrangement of these domains is achieved in specific proteins is a challenging problem. In this issue, Hodgkinson and Kaschka ${ }^{5}$ study transmembrane domains of solute transporters and propose that differences in interdomain hydrophobicity play a role in the organization of transmembrane domains of solute transporters to anchor these proteins to the endoplasmic reticulum.

Protein fold classification is an important step in protein tertiary structure prediction. Many machine learning methods have been applied on this problem. ${ }^{6,7}$ However, most methods do not perform well on low-homology datasets. In this issue, Mohammad and Nagarajaram ${ }^{8}$ tackle this challenge using a SVM-based approach and features derived from predicted secondary structures and predicted burial information of amino acid residues. They report a very competitive $80 \%$ accuracy on proteins that have no more than $25 \%$ sequence identities to proteins in their training dataset.

Analysis of gene expression profiling data has been performed over the past decade and has so far been dominated by applications in disease diagnosis and prognosis. ${ }^{9-11}$ It is thus very refreshing to find in this issue the paper of Li et al. ${ }^{12}$ which links structural fingerprints of drug compounds with expression of particular genes and pathways. Specifically, they have determined the correlation between structural differences of compounds and the expression of a minimal set of feature 
genes. It follows that these feature genes can be utilized as markers to screen for drug candidates from a large number of compounds.

Determining P450 isozyme selectivity of a compound is an important task in drug screening. Large-scale activity data is now available for several P450 isozymes. In this issue, Teramoto and Kato ${ }^{13}$ show how the activity data of one or more P450 isozymes can be used to predict that of another P450 isozyme using ideas from transfer learning. Their algorithm can be a useful tool for P450 selectivity prediction for new chemical entities.

Experiments for the identification of disease-gene association are expensive and have certain limitations. This has led to development of computational techniques for identifying promising genes for experimental verification such as the computational gene knockout procedure of Matsunaga et al. ${ }^{14}$. In this issue, Ochagavia et al. ${ }^{15}$ describes an approach to this challenge that first uses a set of reference genes, with a known association to a disease, to extract candidate genes from molecular interaction networks and pathways. These candidate genes are then prioritized by using multiple methods to evaluate their similarity to the reference genes.

The last two articles in this issue of the Journal of Bioinformatics and Computational Biology are two reviews on simulation in systems biology. In the review by Chignola et al., ${ }^{16}$ the problem of modeling large tumor cell populations and spheroids is presented. They discuss ways to overcome computational limitations. They also present some results on the growth of tumor cells in closed and open environments and of tumor spheroids. In the article of Jørgensen and Haddow, ${ }^{17}$ a large number of simulation tools in systems biology are evaluated in terms of their pedagogical attributes based on the cognitive load theory. They also provide design recommendations for improving these simulation tools from the educational perspective.

\author{
Limsoon Wong \\ Managing Editor
}

\title{
References
}

1. Thuillard M, Moulton V, Identifying and reconstructing lateral transfers from distance matrices by combining the minimum contradiction method and neighbour-net, J Bioinform Comput Biol 9(4):453-470, 2011.

2. Ahmed R, Rangwala H, Karypis G, TOPTMH: Topology predictor for transmembrane alpha helices, J Bioinform Comput Biol 8(1):39-57, 2010.

3. Xu EW, Kearney P, Brown DG, The use of functional domains to improve transmembrane protein topology prediction, J Bioinform Comput Biol 4(1):109-124, 2006.

4. Sadovskaya NS, Sutormin RA, Gelfand MS, Recognition of transmembrane segments in proteins: Review and consistency-based benchmarking of internet servers, J Bioinform Comput Biol 4(5):1033-1056, 2006.

5. Hodgkinson S, Kaschka WP, Patterns of hydrophobicity found in the first and second transmembrane domains of solute transporters suggest a possible role in nascent protein anchoring and organization, J Bioinform Comput Biol 9(4):471-488, 2011. 
6. Chen P, Liu C, Burge L, Mahmood M, Southerland W, Gloster C, Protein fold classification with genetic algorithms and feature selection, J Bioinform Comput Biol 7(5):773-788, 2009.

7. Chinnasamy A, Sung WK, Mittal A, Protein structure and fold prediction using treeaugmented nave Bayesian classifier, J Bioinform Comput Biol 3(4):803-819, 2005.

8. Mohammad TAS, Nagarajaram HA, SVM-based method for protein structural class prediction using secondary structural content and structural information of amino acids, J Bioinform Comput Biol 9(4):489-502, 2011.

9. Licamele L, Getoor L, A method for the detection of meaningful and reproducible group signatures from gene expression profiles, J Bioinform Comput Biol 9(3):431451, 2011.

10. Liu Z, Phan S, Famili F, Pan Y, Lenferink AEG, Cantin C, Collins C, O'ConnorMcCourt M, A multi-strategy approach to informative gene identification from gene expression data, J Bioinform Comput Biol 8(1):19-38, 2010.

11. Zhao Y, Wang G, Additive risk analysis of microarray gene expression data via correlation principal component regression, J Bioinform Comput Biol 8(4):645-659, 2010.

12. Li Y, Tu K, Zheng S, Wang J, Li Y, Hao P, Li X, Association of feature gene expression with structural fingerprints of chemical compounds, J Bioinform Comput Biol 9(4):503-519, 2011.

13. Teramoto R, Kato T, Transfer learning for cytochrome P450 isozyme selectivity prediction, J Bioinform Comput Biol 9(4):521-540, 2011.

14. Matsunaga T, Kuwata S, Muramatsu M, Computational gene knockout reveals transdisease-transgene association structure, J Bioinform Comput Biol 8(5):843-866, 2010 .

15. Ochagavia ME, Miranda J, Nazabal M, Martin A et al., A methodology based on molecular interactions and pathways to find candidate genes associated to diseases: Its application to schizophrenia and Alzheimer's disease, J Bioinform Comput Biol 9(4):541-557, 2011.

16. Chignola R, Fabbro AD, Farina M, Milotti E, Computational challenges of tumor spheroid modeling, J Bioinform Comput Biol 9(4):559-577, 2011.

17. Jørgensen KM, Haddow PC, Visualization in simulation tools: Requirements and a tool specification to support the teaching of dynamic biological processes, J Bioinform Comput Biol 9(4):579-595, 2011. 